\title{
REVISIONES
}

\section{La formación musical en los planes de estudios para maestros de Primaria en la Universidad de Castilla-La Mancha}

\author{
Musical training of curricula for teachers of primary education \\ at the University of Castilla-La Mancha
}

\section{Narciso López García, ${ }^{a}$ Dolores Madrid Vivar, ${ }^{b}$ María del Valle De Moya Martínez}

${ }^{a}$ Grupo de Investigación LABINTIC, Facultad de Educación de Albacete Universidad de Castilla, La Mancha

Telf.: (34) 967213313. Correo electrónico: njlopezg@ gmail.com

bepartamento de Didáctica y Organización Escolar de la Facultad de Ciencias de la Educación de la Universidad de Málaga

Telf.: (34) 952134101. Correo electrónico: 1madrid@uma.es

${ }^{\mathrm{c}}$ Centro de Investigación y Documentación Musical de Castilla, La Mancha Universidad de Castilla, La Mancha

Telf.: (34) 967599200. Correo electrónico: mariavallede.moya@uclm.es

\section{RESUMEN}

El Plan Bolonia ha modificado la estructura de los planes de estudios conducentes a la obtención del título de Maestro definidos en la Ley Orgánica General del Sistema Educativo (LOGSE). Estos cambios han afectado profundamente a las titulaciones de Maestro Especialista, que desaparecen tal y como habían sido concebidas en el RD 1440/1991, del 30 de agosto, pasando a denominarse Menciones Cualificadoras. Nuestra investigación se ha centrado en conocer la estructura actual de la Mención Cualificadora en Educación Musical en el Grado de Maestro de Primaria de la Universidad de Castilla-La Mancha. A su vez, hemos realizado una visión panorámica por la evolución de la formación musical en los diferentes planes de estudios desarrollados en esta Universidad.

Palabras clave: planes de estudio, formación del profesorado, educación musical, Castilla-La Mancha.

\section{ABSTRACT}

The Bologna Process has changed the structure of curricula leading to the award of the title of primary QTS (Qualified Teacher Status) defined in the General Organic Law of the Educational System (LOGSE). These changes have affected the degree of Specialist Teacher, which disappear as it was designed. Our research has focused on understanding the current structure of Qualification Mention in Music Education in the Degree of Teacher of Primary Education from the University of Castilla-La Mancha. At the same time, we have made a vision of the evolution of musical training in different curricula developed at this University.

Key words: studies schedule, teaching education, music education, Castilla-La Mancha. 


\section{INTRODUCCIÓN}

El Espacio Europeo de Educación Superior (EEES) ha modificado sustancialmente el sistema universitario español. Los nuevos planes de estudios para la obtención del Grado de Maestro de Educación Primaria van dirigidos a formar docentes con un perfil generalista, y contemplan las denominadas Menciones Cualificadoras para la formación especializada del profesorado que se deberá hacer cargo de impartir las materias de Educación Física, Educación Musical, Lengua Extranjera, Educación Especial y aquellas otras enseñanzas que, como indica el art. 93 de la LOE (Ley Orgánica 2/2006, de 3 de mayo, de Educación), determine el Gobierno, previa consulta a las comunidades autónomas (López García, 2015).

El Real Decreto 1594/2011, de 4 de noviembre, en su art. 3, establece que será el profesorado del Cuerpo de Maestros con la especialidad de Música el encargado de impartir esta materia, aunque las nuevas directrices educativas definidas en la actual Ley de Educación, LOMCE, (Ley Orgánica 8/2013, de 9 de diciembre, para la mejora de la calidad educativa), han cambiado radicalmente la situación de la Educación Musical en la etapa de Primaria. Este nuevo panorama, que se ha complicado con la reducción de plantillas de profesorado de música en los centros educativos de la mayoría de las comunidades autónomas de nuestro país, ha avivado la hipótesis lanzada años atrás por la doctora Carbajo (2009), cuando afirmaba que una de las consecuencias de este cambio en el perfil profesional del Maestro de Primaria es que, en breve, en los colegios nos podremos encontrar con tres tipos diferentes de docentes en función del grado de preparación musical que posean: por un lado, habrá centros en los que se mantenga el perfil del maestro especialista en Educación Musical; por otro lado, habrá centros con uno o varios maestros con el Grado de Primaria y la Mención en Educación Musical, cuya formación musical específica es muy inferior al grupo anterior; y por último, habrá centros que no dispongan de maestros con los perfiles anteriores, por lo que tendrá que ser uno o varios maestros con el Grado de Primaria los que se tengan que hacer cargo de todo o parte del currículum de esta materia, en base a la formación musical recibida como maestros generalistas. Esta realidad está provocando una sensación de incertidumbre sobre el futuro de la enseñanza de la música en la escuela, así como sobre el papel del maestro generalista frente al del maestro especialista.

En este artículo presentamos un análisis sobre la formación musical general que reciben los futuros maestros de Primaria, así como la formación específica de aquellos alumnos que están estudiando la Mención en Educación Musical en las cuatro Facultades de Educación de la Universidad de Castilla-La Mancha (UCLM), Comunidad Autónoma en la que desarrollamos nuestra labor docente como profesionales de la Educación Musical.

Además, hemos creído fundamental contextualizar nuestro estudio realizando un análisis general de la formación musical incluida en los planes de estudios de la carrera de Maestro de las primeras escuelas normales hasta los programas anteriores a la entrada en vigor del EEES, centrándonos en aquellos que han sido aplicados en la UCLM. De esta manera, hemos obtenido una visión general de la evolución de la enseñanza de la música en los estudios de Maestro que nos ha servido en la elaboración de conclusiones y de prospectivas del futuro a corto y medio plazo de la Educación Musical en la enseñanza primaria. 


\section{PRESENCIA DE LA MÚSICA EN LOS PLANES DE ESTUDIOS DE LA CARRERA DE MAESTRO: ANÁLISIS GENERAL}

Aunque las primeras referencias sobre la enseñanza de la música en los planes de formación de maestros las encontramos en la Real Orden de 24 de agosto de 1878, que establece "por vía de ensayo" y para el curso de 1878-1879, la asignatura de Música y Canto en las Escuelas Normales Centrales de Maestros y Maestras con una duración de, al menos 1 hora diaria, no será hasta el año 1881 para la Escuela Normal Central de Maestras y hasta el año 1898 para la Escuela Normal Central de Maestros, cuando aparezca oficialmente la asignatura de Música en los programas, si bien solo se contemplaba para los estudios de grado superior. De este modo, los Maestros de Grado Elemental quedarían excluidos de recibir estas enseñanzas hasta 1914, año en que se unificarían los Grados Elemental y Superior (Lorenzo, 1995).

Sin embargo, como señala López Casanova (2002), esta formación musical destacará por ser inadecuada e insuficiente y basada, casi en exclusiva, en el aprendizaje de contenidos teóricos muy lejanos de planteamientos prácticos y adaptados a las necesidades pedagógicas y didácticas básicas que requería un sistema educativo falto de tradición musical.

La unificación de Grados establecida por el Plan de 1914, si bien introdujo la formación musical para todos los futuros maestros, la calidad de la enseñanza no mejoró con respecto a los planes anteriores, dedicándose a ofrecer conocimientos de solfeo, teoría de la música y canto sin ninguna finalidad didáctica (Díaz Gómez, 2005).

Será el Plan de 1931 el que potencie enormemente las enseñanzas musicales en la formación del magisterio y proponga un cambio en la estructura académica general de los planes de estudios anteriores, centrándose más en los contenidos de formación profesional que en los puramente culturales (Carbajo, 2009). Este nuevo planteamiento concederá un "trato especial a las materias artísticas y prácticas, considerándolas disciplinas fundamentales en la formación profesional del magisterio" (Cateura, 1992, p. 453).

Los años de Guerra Civil (1936-1939) y el posterior régimen dictatorial resultante (1939-1975) acabaron con las expectativas educativas creadas en la etapa anterior (II República). El nuevo régimen derogó toda normativa relacionada con este período, por lo que se retomaron los planes de estudios para maestros aprobados en 1914 (según Decreto de 10 de febrero de 1940).

La formación musical propuesta en los planes de la dictadura (Plan de 1940 o Plan Bachiller, Plan de 1942 o Plan Provisional y Plan de 1950) se centraría en conocimientos teóricos y solfeísticos de escasa aplicabilidad escolar (Lorenzo, 1995), y en la práctica de cantos religiosos, patrióticos e instructivos como vehículo ideal de transmisión de las ideas del movimiento franquista (Carbajo, 2009).

Tras este período de involución en la formación del profesorado de enseñanza Primaria, el Plan de 1967 presentó unos planteamientos aperturistas y cercanos a las corrientes pedagógicas que llegaban de otros países europeos, en las que se otorgaba especial relevancia a la formación didáctica de las diferentes materias de estudio.

Con respecto a la formación musical de los futuros maestros, esta experimentó un notable avance, poniendo en valor el carácter vivo y activo de la enseñanza de la música y proponiendo una metodología práctica e intuitiva cuyas actividades musicales se deberían desarrollar desde una perspectiva eminentemente didáctica (Lorenzo, 1995).

Este nuevo rumbo quedaría cristalizado con la aplicación de la Ley General de Educación de 1970 y con la publicación, en 1971, del nuevo Plan para las Escuelas de Maestros 
(Plan Experimental). En este, música y su didáctica pasaba a ser una asignatura común a cada una de las cinco especialidades en las que se estructuraban los nuevos estudios de magisterio. Es a partir de este momento cuando los programas de esta disciplina comienzan a proyectarse hacia la escuela primaria, incrementándose el desarrollo y enfoque didáctico de las nuevas metodologías de educación musical (Oriol, 2005).

La Orden de 13 de junio de 1977 sobre directrices para la elaboración de los planes de estudios de las Escuelas Universitarias del Profesorado de Educación General Básica se encargaba de refrendar definitivamente los programas desarrollados en el Plan Experimental, que estarían en vigor hasta la aprobación de la Ley Orgánica 1/1990, de 3 de octubre, de Ordenación General del Sistema Educativo (LOGSE), cuya aplicación conllevaría el establecimiento y publicación de los nuevos planes para maestros en el año 1992.

Tabla 1. Etapas de la formación musical en los estudios de Maestro anteriores al Plan LOGSE

\begin{tabular}{|l|l|}
\hline $\begin{array}{l}\text { Primeros Planes de Estudios } \\
\text { (hasta 1914) }\end{array}$ & $\begin{array}{l}\text { Formación inadecuada e insuficiente. Basada, casi en exclusiva, } \\
\text { en el aprendizaje de contenidos teóricos muy lejanos de } \\
\text { planteamientos prácticos y adaptados a las necesidades } \\
\text { pedagógicas y didácticas básicas. }\end{array}$ \\
\hline Plan de 1931 & $\begin{array}{l}\text { Trato especial a las materias artísticas y prácticas. No se busca } \\
\text { formar profesionales de la música, sino docentes con los } \\
\text { conocimientos técnicos necesarios para que, de forma amena y } \\
\text { eficaz, puedan despertar y desarrollar en el niño el sentimiento } \\
\text { artístico y el amor a la buena tradición lírica española. }\end{array}$ \\
\hline $\begin{array}{l}\text { Primera etapa de la dictadura } \\
\text { franquista (1939-1950) }\end{array}$ & $\begin{array}{l}\text { Cualquier estudio musical previo es convalidable. Carga didáctica } \\
\text { inexistente. }\end{array}$ \\
\hline $\begin{array}{l}\text { Segunda etapa de la dictadura } \\
\text { franquista (1950-1960) }\end{array}$ & $\begin{array}{l}\text { Regulación de los estudios musicales. Formación teórica y } \\
\text { solfeística. Métodos alejados de la práctica escolar. }\end{array}$ \\
\hline $\begin{array}{l}\text { De 1960 a 1992 (últimos años } \\
\text { del franquismo y primeros } \\
\text { años de la democracia) }\end{array}$ & $\begin{array}{l}\text { Giro hacia la didáctica de la música aplicada al aula. Difusión de } \\
\text { las nuevas metodologías de pedagogía musical. }\end{array}$ \\
\hline
\end{tabular}

El art. 16 de la LOGSE establecía que la enseñanza de la música y de las otras especialidades definidas en esta nueva norma serían impartidas por maestros con la especialización correspondiente, por lo que la formación del profesorado se debería ajustar a las necesidades de titulación y de cualificación requeridas por esta nueva ordenación del sistema educativo (Carbajo, 2009).

El Real Decreto 1440/1991, de 30 de agosto, por el que se establece el título universitario, oficial de Maestro, en sus diversas especialidades y las directrices generales propias de los planes de estudios conducentes a su obtención, daba cobertura legal al nuevo panorama establecido por la LOGSE en la formación del magisterio, fijaba la duración y la carga lectiva de los nuevos títulos (carrera universitaria de primer ciclo o de ciclo corto, 
con una duración de 3 años y una carga lectiva no inferior a 180 créditos ni superior a 270), y abría oficialmente un período de tres años para que las universidades, en el ejercicio de su autonomía (según Ley Orgánica de Reforma Universitaria 11/1983, de 25 de agosto), redactasen sus planes de estudios y los remitiesen al Consejo de Universidades para que fueran homologados (Díaz Gómez, 2005).

Los estudios para la obtención del título de Maestro quedaban definidos bajo las siguientes denominaciones:

- $\quad$ Título de Maestro - Especialidad de Educación Infantil.

- Título de Maestro - Especialidad de Educación Primaria.

- Título de Maestro - Especialidad de Lengua Extranjera.

- Título de Maestro - Especialidad de Educación Física.

- Título de Maestro - Especialidad de Educación Musical.

- Título de Maestro - Especialidad de Educación Especial.

- Título de Maestro - Especialidad de Audición y Lenguaje.

En estos nuevos programas, la formación musical de los futuros maestros de primaria quedaba recogida como Materia Troncal de la Especialidad en todas las titulaciones, excepto en la de Audición y Lenguaje. Asimismo, se articulaba, por primera vez en la historia de la enseñanza de la música en nuestro país, una titulación específica para el profesorado de Educación Musical (Lorenzo, 1995).

Este nuevo sistema se ponía en marcha durante el curso 1992-1993, por lo que las primeras promociones de titulados terminaron sus estudios en el curso 1994-1995.

\section{LA FORMACIÓN MUSICAL DE LOS FUTUROS MAESTROS EN LOS PLANES DE ESTUDIOS DE LA UCLM}

Aunque la Universidad de Castilla-La Mancha no comenzaría su andadura hasta el curso 1985-1986 (el 10 de julio de 1982 se publicaba en el BOE la Ley 27/1982, de 30 de junio, por la que se crea la UCLM, inaugurándose el primer curso el 4 de octubre de 1985), las escuelas de maestros de las cinco capitales de provincia de la región fueron creadas, como tantas otras, a partir de 1839.

Sin embargo, los datos proporcionados por Infante (2010) y Belmonte (2010) nos confirman que los planes de estudios de las normales en el siglo XIX fueron decretados con total uniformidad desde el Ministerio de Instrucción Pública y que, ya en el siglo XX, no será hasta la publicación de la LRU (Ley Orgánica de Reforma Universitaria 11/1983) cuando las universidades españolas gocen de cierta autonomía y libertad curricular.

En el curso 1985-1986 las Escuelas Universitarias de Formación del Profesorado de EGB de Castilla-La Mancha (denominación aprobada según Decreto 1383/1972 de 25 de mayo), a excepción de la Escuela de Guadalajara, adscrita a la Universidad de Alcalá de Henares, comenzaban su andadura como centros dependientes de la UCLM. Siguiendo las directrices marcadas por la Orden de 13 de junio de 1977 sobre la elaboración de los planes de estudio de las Escuelas Universitarias del Profesorado de Educación General Básica, y haciendo uso de la autonomía tutelada que la LRU les proporcionaba, las escuelas castellano-manchegas diseñaron sus propios programas que se mantendrían hasta el año 
1992, momento en que se comenzarían a aplicar los nuevos planes de estudios surgidos como consecuencia de la entrada en vigor de la LOGSE.

En estos años, la formación musical en los estudios de magisterio de nuestra región era muy similar a la ofrecida en planes anteriores, especialmente en los planes surgidos a partir de 1914, sin olvidar el perfil técnico-práctico que el Plan de 1967 había introducido a esta titulación. Teniendo en cuenta que estos programas formaban a maestros generalistas, la Educación Musical no iba más allá de ser una asignatura cuya estructura curricular se fundamentaba en dar a conocer a los estudiantes los contenidos básicos sobre lenguaje musical, teoría de la música e historia de la música, así como las nociones básicas para interpretar sencillas melodías con un instrumento melódico (generalmente la flauta dulce), por lo que la preparación musical de los egresados no terminaba de capacitarlos debidamente para impartir esta enseñanza en las escuelas con las garantías suficientes.

Tabla 2. Asignaturas de Educación Musical cursadas en las escuelas de magisterio de la UCLM entre 1977 y 1992

\begin{tabular}{|c|c|c|c|c|c|}
\hline \multicolumn{6}{|c|}{ Formación Musical en la UCLM - Plan de Estudios de 1977 (vigente hasta 1992) } \\
\hline & Curso & Asignatura & Especialidades & Tipo & Créditos \\
\hline \multirow{4}{*}{$\begin{array}{l}\text { Escuela de } \\
\text { Magisterio } \\
\text { de Albacete }\end{array}$} & $1^{\circ}$ & \multicolumn{4}{|c|}{ No se cursaba ninguna asignatura relacionada con la Educación Musical } \\
\hline & $2^{\circ}$ & Música y su Didáctica & Todas* & OB. & 12 \\
\hline & $3^{\circ}$ & $\begin{array}{c}\text { Educación Artística Musical del } \\
\text { Preescolar }\end{array}$ & Preescolar & OB. & 9 \\
\hline & $3^{\circ}$ & $\begin{array}{l}\text { Ampliación de Música y su } \\
\text { Didáctica** }\end{array}$ & CC. Humanas & OPT. & 4,5 \\
\hline \multirow{5}{*}{$\begin{array}{c}\text { Escuela de } \\
\text { Magisterio } \\
\text { de Ciudad } \\
\text { Real }\end{array}$} & $1^{\circ}$ & \multicolumn{4}{|c|}{ No se cursaba ninguna asignatura relacionada con la Educación Musical } \\
\hline & $2^{\circ}$ & Música I & Todas* & OB. & 9 \\
\hline & $2^{\circ}$ & $\begin{array}{c}\text { La Guitarra y su Aplicación } \\
\text { Didáctica en EGB }\end{array}$ & Todas* & OPT. & 4,5 \\
\hline & $3^{\circ}$ & Música II & $\begin{array}{c}\text { CC. Humanas, } \\
\text { Ciencias y } \\
\text { Filología }\end{array}$ & OB. & 6 \\
\hline & $3^{\circ}$ & $\begin{array}{l}\text { Música y su Didáctica en } \\
\text { Preescolar }\end{array}$ & Preescolar & OB. & 6 \\
\hline \multirow{3}{*}{$\begin{array}{l}\text { Escuela de } \\
\text { Magisterio } \\
\text { de Cuenca }\end{array}$} & $1^{\mathrm{o}}$ & Música y su Didáctica & Todas* & OB. & 9 \\
\hline & $2^{\circ}$ & Música para la Edad Preescolar & Preescolar & OB. & 9 \\
\hline & $3^{\circ}$ & $\begin{array}{l}\text { Didáctica de la Música para la } \\
\text { Educación Especial }\end{array}$ & Ed. Especial & OB. & 6 \\
\hline \multirow{3}{*}{$\begin{array}{l}\text { Escuela de } \\
\text { Magisterio } \\
\text { de Toledo }\end{array}$} & $1^{\circ}$ & Música I & Todas* & OB. & 9 \\
\hline & $2^{\circ}$ & Música II & Todas* & OB. & 9 \\
\hline & $3^{\circ}$ & Educación Musical en Preescolar & Preescolar & OB. & 9 \\
\hline
\end{tabular}

* CC. Humanas, Ciencias, Filología y Preescolar (en Cuenca también Ed. Especial). ** A partir del curso 88-89. 


\subsection{LOS PLANES LOGSE Y LA NUEVA ESPECIALIDAD DE MAESTRO EN EDUCACIÓN MUSICAL}

Tras la publicación de la LOGSE se producía una de las situaciones de cambio más importantes en la historia de la formación del magisterio. La nueva configuración del sistema educativo español requería unos nuevos planes de estudios estructurados en siete especialidades, en los que el perfil de Maestro Generalista quedaba relegado a un segundo plano, dando paso a los títulos con especialización, adaptándose, así, a las áreas curriculares estipuladas en la nueva Ley.

Fruto del trabajo de los movimientos estudiantiles, el título de Maestro Especialista en Educación Musical quedaba implantado en los cuatro centros de formación del profesorado de primaria y, aunque la estructura general del plan de estudios era la misma para cada escuela, la organización curricular y las asignaturas para la obtención de créditos de libre configuración estaban abiertas a las necesidades de cada una de ellas, según los recursos profesionales y materiales de que dispusieran.

El BOE del 21 de octubre de 1992 publicaba la Resolución 23487 de 30 de septiembre de 1992, de la Universidad de Castilla-La Mancha, por la que se hace público el Plan de Estudios del título de Maestro, especialidad de Educación Musical, de las Escuelas Universitarias de Formación del Profesorado de EGB de esta Universidad. En este plan se incluyen las asignaturas optativas propuestas por la UCLM y los créditos de libre configuración que el alumno deberá conseguir para complementar y completar su formación. La carga lectiva global para la obtención de la titulación de Maestro Especialista en Educación Musical será de 206 créditos.

Además, el resto de especialidades, también la Especialidad de Audición y Lenguaje -las directrices generales publicadas en el RD1440/1991 no hacían referencia alguna a la formación musical en esta titulación- ofertaron diferentes asignaturas relacionadas con esta área de conocimiento.

Tabla 3. Asignaturas del resto de especialidades relacionadas con la Educación Musical, Planes de Estudios de 1992

\begin{tabular}{|c|c|c|c|c|}
\hline & Asignatura & Tipo & Curso & Créditos \\
\hline \multirow{2}{*}{$\begin{array}{c}\text { Especialidad de } \\
\text { Audición y } \\
\text { Lenguaje }\end{array}$} & Didáctica de la Expresión Musical & OBU & $2^{\circ}$ & 4,5 \\
\cline { 2 - 5 } & Formación Vocal y Auditiva & OP & $1^{\circ}$ & 4,5 \\
\cline { 2 - 5 } & Formación Rítmica y Danza & OP & $2^{\circ}$ & 4,5 \\
\hline $\begin{array}{c}\text { Especialidad de } \\
\text { Educación Especial }\end{array}$ & Didáctica de la Expresión Musical & T & $2^{\circ}$ & 4,5 \\
\hline \multirow{2}{*}{$\begin{array}{c}\text { Especialidad de } \\
\text { Educación Física }\end{array}$} & Didáctica de la Expresión Musical & T & $2^{\circ}$ & 3 \\
\cline { 2 - 5 } & Danzas Populares (solo en Ciudad Real) & OP & $3^{\circ}$ & 3 \\
\cline { 2 - 5 } & Formación Rítmica y Danza (solo en Toledo) & OP & $3^{\circ}$ & 4,5 \\
\cline { 2 - 5 } & Formación Rítmica y Danza (solo en Albacete) & OP & $2^{\circ}$ & 4,5 \\
\hline
\end{tabular}


Estudios Pedagógicos XLIII, N 1: 423-438, 2017

LA FORMACIÓN MUSICAL EN LOS PLANES DE ESTUDIOS PARA MAESTROS DE PRIMARIA EN LA UNIVERSIDAD DE CASTILLA-LA MANCHA

\begin{tabular}{|c|c|c|c|c|}
\hline $\begin{array}{c}\text { Especialidad de } \\
\text { Lenguas Extranjeras }\end{array}$ & Educación Artística y su Didáctica & $\mathrm{T}$ & $2^{\circ}$ & $6(3+3)$ \\
\hline $\begin{array}{c}\text { Especialidad de } \\
\text { Educación Primaria }\end{array}$ & Didáctica de la Expresión Musical & $\mathrm{T}$ & $1^{\circ}$ & 4,5 \\
\cline { 2 - 6 } & Formación Vocal y Auditiva (solo en Albacete) & $\mathrm{OP}$ & $3^{\circ}$ & 4,5 \\
\hline \multirow{2}{*}{$\begin{array}{c}\text { Especialidad de } \\
\text { Educación Infantil }\end{array}$} & Didáctica de la Expresión Musical I & $\mathrm{T}$ & $1^{\circ}$ & 6 \\
\cline { 2 - 6 } & Didáctica de la Expresión Musical II & OBU & $2^{\circ}$ & 6 \\
\cline { 2 - 6 } & Formación Vocal y Auditiva (solo en Albacete) & OP & $3^{\circ}$ & 4,5 \\
\hline T: Materia TRONCAL / OBU: Materia OBLIGATORIA DE UNIVERSIDAD / OP: Materia \\
OPTATIVA
\end{tabular}

El 29 de octubre de 1999 aparecía en el BOE la Resolución de 1 de octubre de la UCLM por la que se hacía pública la modificación a los planes de estudios del título de Maestro en sus siete especialidades aprobados en 1992, reforma que intentaba solucionar los desajustes de los programas anteriores. En este nuevo plan se aumentaba la carga lectiva total correspondiente a las asignaturas troncales y obligatorias relacionadas con la formación musical, pasando de 57 créditos a 60 créditos. A su vez, la oferta de asignaturas optativas específicas se ampliaba en las cuatro escuelas, sin embargo, la carga lectiva global de las asignaturas relacionadas con la formación musical en el resto de especialidades se reducía en 7,5 créditos ( 3 créditos en la Especialidad de Educación Física y 4,5 créditos en la Especialidad de Educación Primaria).

Este plan de estudios se mantuvo vigente hasta el curso académico 2008-2009. Tras la publicación en el BOE de la Resolución de 17 de diciembre de 2007, sobre la adecuación de planes de estudios conducentes a la obtención del título de Grado para el ejercicio de la profesión de Maestro y de la Orden de 27 de diciembre de 2007, sobre la verificación de los títulos universitarios oficiales, los distintos departamentos de las cuatro escuelas de magisterio de la UCLM comenzaron a trabajar para adecuar sus planes al nuevo Espacio Europeo de Educación Superior.

\subsection{LA SITUACIÓN ACTUAL DE LA FORMACIÓN MUSICAL EN LOS PROGRAMAS PARA LA OBTENCIÓN DE TÍTULO DE GRADUADO EN MAESTRO DE EDUCACIÓN PRIMARIA EN LA UCLM}

El denominado Proceso de Bolonia, del que surgirá el Espacio Europeo de Educación Superior, nacía con la intención de adaptar las titulaciones universitarias de 47 países europeos a la nueva realidad social en la que vivimos (Montero, 2010). Uno de los cambios más significativos ha sido la adopción del Sistema Europeo de Transferencia y Acumulación de Créditos (European Credit Transfer and Accumulation System-ECTS), sistema que permite medir el trabajo que deben realizar los estudiantes para la adquisición de competencias, conocimientos, capacidades y destrezas necesarias para superar las diferentes materias de su plan de estudios.

Este nuevo sistema de créditos tiene como objetivo principal facilitar la movilidad de los estudiantes, dinamizar la adquisición del conocimiento, incorporar las TIC a los 
procesos de enseñanza-aprendizaje y ofrecer autonomía al estudiante en sus tareas. La actividad de estudio (entre 25 y 30 horas por crédito) incluye el tiempo dedicado a las horas lectivas, horas de estudio, tutorías, seminarios, prácticas, trabajos o proyectos, así como las horas necesarias para la preparación y realización de exámenes y evaluaciones (López García, 2015).

En España, la adecuación de las titulaciones al EEES comenzó con el análisis comparativo de las titulaciones antiguas con las de otros países europeos. Para ello, el Consejo de Ministros de 19 de julio de 2002 acordaba crear la ANECA $^{1}$, quien encargó a los decanos de las facultades de educación y directores de las escuelas universitarias de magisterio (la denominada Red de Magisterio, que comenzará a trabajar en 2003), la elaboración de un proyecto de adecuación de las titulaciones de maestro al EEES. A la vista de los informes realizados por la Red de Magisterio, el planteamiento inicial proponía una reestructuración de las antiguas diplomaturas en dos grados: Grado de Maestro en Educación Infantil y Grado de Maestro en Educación Primaria. Asimismo, para encajar las diversas especialidades surgidas con la aplicación de la LOGSE en el nuevo diseño, se propuso que el Grado de Primaria se completase con la elección de un itinerario formativo relacionado con la Lengua Extranjera, la Educación Física, la Educación Especial o la Educación Musical, que han terminado denominándose Menciones Cualificadoras (Carbajo, 2009).

El trabajo llevado a cabo por la Red de Magisterio quedó recogido en el Libro Blanco sobre el Título de Grado en Magisterio, publicado en 2005. En este se establece que la formación de los futuros Maestros tendrá una carga lectiva de 240 créditos ECTS que deberán cursarse en cuatro cursos. Estos nuevos grados se caracterizarán por su perfil generalista y por estar estructurados en tres módulos diseñados por competencias, cuya carga lectiva total será de 210 créditos ECTS, quedando 30 créditos a criterio de las universidades para que, en virtud de su autonomía, sean asignados como consideren (Gutiérrez, Luengo \& Casas, 2011).

Los primeros pasos dados por las universidades españolas hacia la convergencia europea en materia de educación superior quedaban plasmados en la Ley Orgánica 6/2001, de 21 de diciembre, de Universidades (LOU), pero será a partir de 2007 cuando estas se pongan a trabajar en la modificación de sus planes de estudios para tenerlos preparados dentro de los plazos establecidos.

La UCLM obtenía la verificación de los planes de estudios de Graduado en Maestro en Educación Primaria y de Graduado en Maestro en Educación Infantil por el Consejo de Universidades y previo informe positivo de la ANECA, el 4 de diciembre de 2009. A su vez, mediante las Resoluciones 3587 y 3588, de 17 de febrero de 2010 de la UCLM, se hacía pública la nueva ordenación académica para estas titulaciones. Además, estas dos Resoluciones disponen que cada una de las cuatro facultades tendrá su propia oferta de asignaturas optativas y menciones.

La ANECA (Agencia Nacional de Evaluación de la Calidad y Acreditación) es un organismo autónomo, adscrito al Ministerio de Educación, Cultura y Deporte de España, cuyo objetivo fundamental es contribuir a la mejora de la calidad del sistema de educación superior mediante la evaluación, certificación y acreditación de enseñanzas, profesorado e instituciones. 
Tabla 4. Carga lectiva global del título de Graduado en Maestro en Educación Primaria en la UCLM

\begin{tabular}{|c|c|c|c|c|c|}
\hline \multicolumn{6}{|c|}{ Crado en Maestro en Educación Primaria } \\
\hline Curso & $\begin{array}{c}\text { Formación } \\
\text { Básica }\end{array}$ & Obligatorias & Optativas & Prácticas Externas & $\begin{array}{c}\text { Trabajo de Fin } \\
\text { de Grado }\end{array}$ \\
\hline $1^{\text {o }}$ & 36 & 24 & 0 & 0 & 0 \\
\hline $2^{\text {o }}$ & 24 & 36 & 0 & 0 & 0 \\
\hline $3^{\text {o }}$ & 0 & 24 & 12 & 24 & 0 \\
\hline $4^{\text {o }}$ & 0 & 18 & 12 & 24 & 6 \\
\hline Total & 60 & 102 & 24 & 48 & 6 \\
\hline
\end{tabular}

En estos nuevos planes se establece una formación musical básica tanto para los estudiantes del Grado de Educación Infantil como para los del Grado de Educación Primaria.

Tabla 5. Formación musical básica para los Grados en Ed. Infantil y en Ed. Primaria en la UCLM

\begin{tabular}{|l|c|c|c|c|}
\hline & Asignatura & Tipo & Créditos ECTS & Curso \\
\hline \multirow{2}{*}{ Grado en Educación Infantil } & Percepción y Expresión Musical & OB & 6 & $3^{\circ}$ \\
\cline { 2 - 5 } & Expresión Artística & OB & $3+3$ & $4^{\circ}$ \\
\hline Grado en Educación Primaria & Educación Musical & OB & 6 & $3^{\circ}$ \\
\hline
\end{tabular}

La Educación Musical, por lo tanto, forma parte de las competencias básicas que debe haber adquirido cualquier maestro al término de sus estudios. Las competencias relacionadas con la Educación Musical, Plástica y Visual quedan explicitadas en la Orden ECI/3857/2007 en los siguientes términos:

- Comprender los principios que contribuyen a la formación cultural, personal y social desde las artes.

- Conocer el currículo escolar de la educación artística, en sus aspectos plásticos, audiovisual y musical.

- Adquirir recursos para fomentar la participación a lo largo de la vida en actividades musicales y plásticas dentro y fuera de la escuela.

- Desarrollar y evaluar contenidos del currículo mediante recursos didácticos apropiados y promover las competencias correspondientes en los estudiantes. 


\subsection{LA ESTRUCTURA ACTUAL DE LA MENCIÓN CUALIFICADORA EN EDUCACIÓN MUSICAL EN LAS FACULTADES DE EDUCACIÓN DE LA UCLM}

El Reglamento para el diseño, elaboración y aprobación de los planes de estudios de grado, aprobado en Consejo de Gobierno de la UCLM, de 17 de abril de 2008, de acuerdo con la LOU y con los Estatutos de la UCLM, establecía que la elaboración y modificación de los planes de estudios conducentes a la obtención del título de Maestro correspondía a las escuelas de magisterio, con la participación de sus propios departamentos. A su vez, el Reglamento especificaba que estos planes de estudios debían ser fruto de un consenso generalizado en el que todas las partes implicadas expresaran sus opiniones, y que los mismos debían mantenerse dentro del marco definido por el RD 1393/2007, de 29 de octubre, por el que se establece la ordenación de las enseñanzas universitarias oficiales.

La existencia del título de Maestro Especialista en Educación Musical (planes 1992 y 1999) en las cuatro escuelas de magisterio y, por tanto, la existencia de los recursos humanos y materiales necesarios para su desarrollo académico, favoreció la adaptación de los antiguos planes de estudios a las nuevas Menciones Cualificadoras en Educación Musical, por lo que las cuatro facultades de educación optaron por ofertarla.

Aunque las bases de los nuevos currículos de la Mención en Educación Musical mantenían una ordenación similar, que se ceñía a los requisitos establecidos por el RD $1393 / 2007$ y por la Orden ECI/3857/2007, por la que se establecen los requisitos para la verificación de los títulos universitarios oficiales que habiliten para el ejercicio de la profesión de Maestro en Educación Primaria, los centros dispusieron de un importante margen de autonomía que les permitió actuar en base a los recursos materiales y profesionales de que disponían.

Atendiendo a las indicaciones finales de la Resolución 3588, de 17 de febrero de 2010 de la UCLM sobre la configuración de menciones y asignaturas optativas, cada una de las cuatro facultades de educación propuso la ordenación de sus menciones y las asignaturas que conformarían dichas menciones. Meses después, la ANECA hacía pública la evaluación positiva de los planes de estudios de todos los títulos de grado y de máster presentados por la UCLM, confirmando que estos cumplían los requisitos necesarios para ser impartidos en los distintos campus y que cumplían con el modelo establecido por la Unión Europea.

La siguiente tabla recoge las asignaturas propias de la Mención Cualificadora en Educación Musical de cada una de las facultades de educación de la UCLM. 
Tabla 6. Aspectos generales de las asignaturas de la Mención en Educación Musical de la UCLM

\begin{tabular}{|c|c|c|c|c|}
\hline \multicolumn{5}{|c|}{ Mención Cualificadora en Educación Musical } \\
\hline Asignatura & Curso & ECTS & Duración & Tipo \\
\hline \multicolumn{5}{|c|}{ Facultad de Educación de Albacete } \\
\hline Didáctica de la Música & $3^{\circ}$ & 6 & $\mathrm{C} 1$ & $\mathrm{PE}$ \\
\hline Historia de la Música & $3^{\circ}$ & 6 & $\mathrm{C} 2$ & $\mathrm{OP}$ \\
\hline Expresión Vocal y Corporal & $4^{\circ}$ & 4,5 & $\mathrm{C} 1$ & OP \\
\hline Agrupaciones Musicales & $4^{\circ}$ & 4,5 & $\mathrm{C} 1$ & $\mathrm{OP}$ \\
\hline Lenguaje Musical y Armonía & $4^{\circ}$ & 4,5 & $\mathrm{C} 1$ & OP \\
\hline Nuevas Tecnologías y Tendencias Musicales & $4^{\circ}$ & 4,5 & $\mathrm{C} 1$ & $\mathrm{OP}$ \\
\hline \multicolumn{5}{|c|}{ Facultad de Educación de Ciudad Real } \\
\hline Lenguajes Musicales & $3^{\circ}$ & 6 & $\mathrm{C} 1$ & $\mathrm{PE}$ \\
\hline Expresión Instrumental & $3^{\circ}$ & 6 & $\mathrm{C} 2$ & $\mathrm{OP}$ \\
\hline Didáctica de la Música & $4^{\circ}$ & 4,5 & $\mathrm{C} 1$ & $\mathrm{OP}$ \\
\hline Historia de la Música & $4^{\circ}$ & 4,5 & $\mathrm{C} 1$ & OP \\
\hline Prácticas Musicales Creativas & $4^{\circ}$ & 4,5 & $\mathrm{C} 1$ & $\mathrm{OP}$ \\
\hline Expresión Vocal y Corporal & $4^{\circ}$ & 4,5 & $\mathrm{C} 1$ & $\mathrm{OP}$ \\
\hline \multicolumn{5}{|c|}{ Facultad de Educación de Cuenca } \\
\hline Lenguajes Musicales & $3^{\circ}$ & 6 & $\mathrm{C} 1$ & $\mathrm{PE}$ \\
\hline Expresión Instrumental & $3^{\circ}$ & 6 & $\mathrm{C} 2$ & $\mathrm{OP}$ \\
\hline Expresión Vocal y Corporal & $4^{\circ}$ & 4,5 & $\mathrm{C} 1$ & $\mathrm{OP}$ \\
\hline Historia de la Música & $4^{\circ}$ & 4,5 & $\mathrm{C} 1$ & $\mathrm{OP}$ \\
\hline Didáctica de la Música & $4^{\circ}$ & 4,5 & $\mathrm{C} 1$ & $\mathrm{OP}$ \\
\hline Prácticas Musicales Creativas & $4^{\circ}$ & 4,5 & $\mathrm{C} 1$ & $\mathrm{OP}$ \\
\hline \multicolumn{5}{|c|}{ Facultad de Educación de Toledo } \\
\hline Lenguas Musicales & $3^{\circ}$ & 6 & $\mathrm{C} 1$ & $\mathrm{PE}$ \\
\hline Expresión Instrumental & $3^{\circ}$ & 6 & $\mathrm{C} 2$ & $\mathrm{OP}$ \\
\hline Expresión Vocal y Corporal & $4^{\circ}$ & 4,5 & $\mathrm{C} 1$ & $\mathrm{OP}$ \\
\hline Historia de la Música & $4^{\circ}$ & 4,5 & $\mathrm{C} 1$ & OP \\
\hline Didáctica de la Música & $4^{\circ}$ & 4,5 & $\mathrm{C} 1$ & OP \\
\hline Prácticas Musicales Creativas & $4^{\circ}$ & 4,5 & $\mathrm{C} 1$ & OP \\
\hline
\end{tabular}

PE: Prácticas Externas / OP: Asignatura Optativa de Mención / C1: Primer Cuatrimestre / C2: Segundo Cuatrimestre 
Como se puede observar, la denominación propuesta para las asignaturas en cada una de las facultades es similar. En cuanto al tipo de asignaturas, la carga lectiva, el curso y el cuatrimestre en que deberán ser impartidas, es común para los cuatro campus.

Tabla 7. Competencias específicas del Maestro de Primaria con Mención en Educación Musical

\begin{tabular}{|c|}
\hline Competencias docentes específicas del perfil de Maestro de Educación Musical \\
\hline Conocimientos Disciplinares (SABER) \\
\hline $\begin{array}{l}\text { Conocer la dimensión musical de las áreas del conocimiento: derivaciones de la acústica en la } \\
\text { Educación Musical }\end{array}$ \\
\hline $\begin{array}{l}\text { Conocer los principios de neurofisiología sobre estimulación y percepción sonora, semiótica y } \\
\text { simbolización en los lenguajes musicales }\end{array}$ \\
\hline $\begin{array}{l}\text { Conocer los fundamentos y desarrollo de la didáctica y la pedagogía musicales y ser capaz de } \\
\text { realizar adaptaciones que permitan acceder a todos los niños al disfrute de la música y a su uso } \\
\text { como medio de expresión }\end{array}$ \\
\hline $\begin{array}{l}\text { Ser capaz de recurrir al uso de las nuevas tecnologías, tanto el almacenamiento, grabación y edición } \\
\text { a nivel educativo }\end{array}$ \\
\hline $\begin{array}{l}\text { Ser capaz de analizar las corrientes de educación musical actuales, extrayendo conceptos y líneas } \\
\text { metodológicas con una coherencia sistemática }\end{array}$ \\
\hline Buscar y utilizar bibliografía y materiales de apoyo en, al menos, dos lenguas \\
\hline Competencias Profesionales (SABER HACER) \\
\hline Saber utilizar el juego musical como elemento didáctico y como contenido \\
\hline Capacidad de utilizar referencias variadas para improvisar solo o en grupo \\
\hline $\begin{array}{l}\text { Tomar conciencia de los elementos temáticos desde un análisis perceptivo de la escucha, elaborando } \\
\text { documentos, esquemas y partituras }\end{array}$ \\
\hline Promover la comprensión de las formas estéticas contemporáneas, tonales y atonales \\
\hline $\begin{array}{l}\text { Dominar la didáctica específica de la Ed. Musical, así como las técnicas de programación, diseño } \\
\text { de sesiones, elección y creación de recursos, así como estrategias de intervención }\end{array}$ \\
\hline $\begin{array}{l}\text { Conocer los fundamentos de la cultura popular, con especial referencia al folklore propios de la } \\
\text { localidad y la Comunidad Autónoma }\end{array}$ \\
\hline $\begin{array}{l}\text { Conocer y dominar los principios de la expresión y la comunicación corporal más directamente } \\
\text { relacionados con el hecho musical y con la danza }\end{array}$ \\
\hline $\begin{array}{l}\text { Conocer los fundamentos del lenguaje musical, técnica instrumental y vocal, armonía, rítmica y } \\
\text { danza }\end{array}$ \\
\hline Ser capaz de organizar y dirigir una agrupación instrumental o coral infantil \\
\hline Competencia Académicas \\
\hline Conocer las manifestaciones musicales de las diferentes culturas \\
\hline Conocer, valorar y seleccionar obras musicales de referencia de todos los estilos, tiempos y culturas \\
\hline Conocer las técnicas de representación del lenguaje musical \\
\hline
\end{tabular}




\subsection{EL MÁSTER SEMI-PRESENCIAL EN INNOVACIÓN E INVESTIGACIÓN MUSICAL DE LA UCLM}

El 22 de julio de 2014 se firmaba el Acuerdo del Consejo de Gobierno de la UCLM, por el que se aprueba la propuesta de Máster en Innovación e Investigación Musical (MIMU-1). Este Máster, vinculado al Centro de Investigación y Documentación Musical de CastillaLa Mancha (Unidad asociada al CSIC), va dirigido a profesionales de la música, tanto en el ámbito de la innovación e investigación musicológica como educativa (maestros y profesores de Música de enseñanza secundaria, conservatorios y escuelas de música, titulados en Grado Superior de Conservatorio, en Grado de Musicología o Historia y Ciencias de la Música, Educación, Humanidades, Arte, Historia, Filología o Bellas Artes con conocimientos musicales y profesionales de la música con titulación superior) y se plantea los siguientes objetivos fundamentales:

- Capacitar a los estudiantes en metodologías, competencias y paradigmas de investigación relacionados con el patrimonio musical, con los procesos de análisis y edición musical, la contextualización del fenómeno musical y su convivencia con otras expresiones artísticas de otros ámbitos artísticos.

- Dar a conocer los nuevos paradigmas en investigación educativa, tanto desde la perspectiva de la percepción como de la expresión, profundizando en las metodologías creativas, así como en la reflexión sobre la investigación en nuevas tecnologías, músicas urbanas y medios audiovisuales.

Tabla 8. Áreas de Conocimiento y carga lectiva del MIMU-1 ( $1^{\mathrm{a}}$ Ed.)

\begin{tabular}{|l|c|}
\hline \multicolumn{1}{|c|}{ Área de Conocimiento } & ECTS \\
\hline Patrimonio Musical & $6 \mathrm{~T}+2 \mathrm{P}$ \\
\hline Edición y Análisis Musical & $6 \mathrm{~T}+2 \mathrm{P}$ \\
\hline Estudios Interdisciplinares: Música, Arte y Literatura & $6 \mathrm{~T}+2 \mathrm{P}$ \\
\hline Educación Musical & $6 \mathrm{~T}+2 \mathrm{P}$ \\
\hline Música y TIC & $6 \mathrm{~T}+2 \mathrm{P}$ \\
\hline Músicas Urbanas y Medios Audiovisuales & $6 \mathrm{~T}+2 \mathrm{P}$ \\
\hline Trabajo de Fin de Máster & $12 \mathrm{~T}$ \\
\hline \multicolumn{2}{|c|}{ Número total de créditos ECTS $=60(48 \mathrm{~T}+12 \mathrm{P})$} \\
\hline \multicolumn{2}{|c|}{$\mathrm{T}:$ Teóricos / P: Prácticos } \\
\hline
\end{tabular}

El 25 de marzo de 2015 se autorizaba la continuidad del Máster (MIMU - II) para el curso académico 2015-2016, manteniendo la estructura del curso anterior. 
La coordinación y los seminarios presenciales se están realizando desde la Facultad de Educación de Ciudad Real, siendo impartido por profesorado especializado de la UCLM y de otras universidades españolas.

\section{CONCLUSIONES}

La evolución histórica de la Educación Musical en los procesos formativos de los futuros maestros en España nos ha mostrado las grandes carencias de esta área de conocimiento en los programas oficiales.

No será hasta la entrada en vigor de la LOGSE, y con ella la incorporación de la música como asignatura obligatoria en los currículos de Primaria, cuando se produzca un profundo cambio en los planes de estudios para la obtención del título de Maestro, con la aparición del título específico de Maestro Especialista en Educación Musical; el papel de Maestro Generalista quedaba en un segundo plano cediendo terreno al de Maestro Especialista.

Entre 1992 y 2008 se pusieron en marcha dos programas diferentes, aunque con una estructura similar, en los que las asignaturas propias de la especialización sumaban un alto porcentaje de la carga lectiva total. De esta manera, se buscaba un perfil de Maestro de Música muy concreto, capaz de reunir una serie de características relacionadas con sus capacidades como educador con conocimientos psicológicos, pedagógicos y sociológicos a nivel musical en el ámbito de la educación.

La adecuación de los títulos de Maestro al Espacio Europeo de Educación Superior ha supuesto un importante cambio en la estructura de los planes de estudios actuales, especialmente en lo que se refiere a la configuración de las especialidades implantadas por la LOGSE. El perfil de Maestro Generalista ha vuelto a ocupar un papel fundamental, mientras que las antiguas especialidades han perdido peso específico pasando a denominarse menciones cualificadoras. Esto ha supuesto una drástica reducción de créditos ECTS relacionados con la formación musical específica y, por tanto, la desaparición de un buen número de asignaturas propias de la especialidad.

Esta nueva situación, junto con la puesta en marcha de la nueva Ley de Educación (LOMCE), está generando una serie de dudas relacionadas, por un lado, con la preparación de los futuros Maestros de Música y, por otro, con la coexistencia de diferentes perfiles docentes en los centros que podrán realizar las labores de Especialista de Educación Musical.

La convocatoria de oposiciones al Cuerpo de Maestros publicada por la Junta de Comunidades de Castilla-La Mancha para los meses de junio y julio de 2016, abrirá las puertas a las primeras promociones de graduados en Maestro de Educación Primaria con su correspondiente Mención Cualificadora en Educación Musical, por lo que a finales del curso escolar 2016-2017 tendremos los primeros datos relacionados con las competencias musicales adquiridas por estos primeros egresados. Será a partir de este momento cuando podamos hacer valoraciones fundamentadas sobre los nuevos grados.

Para concluir, sirvan como reflexión las siguientes palabras de Aróstegui (2010, p. 189) sobre el perfil deseado del futuro Maestro de Música para la escuela primaria: "Necesitamos maestros de música, no músicos educadores que, siguiendo con el juego de palabras, desarrollen en el aula de enseñanzas obligatorias una música educativa antes que una educación musical". 


\section{REFERENCIAS BIBLIOGRÁFICAS}

Aróstegui, J.L. (2010). Formación del profesorado de música: planes de estudio en Europa y América Latina. Profesorado. Revista de Currículum y Formación del Profesorado, 2(14), 3-7.

Belmonte, F. (2010). La Escuela Normal de Maestros de Albacete (1842-1900). Cuenca: Ediciones de la Universidad de Castilla-La Mancha.

Carbajo, C. (2009). El perfil del docente de música en educación primaria: autopercepción de competencias profesionales y la práctica de aula (Tesis doctoral sin publicar). Universidad de Murcia: Departamento de Teoría e Historia de la Educación.

Cateura, M. (1992). Por una educación musical en España: estudio comparativo con otros países. Barcelona: Promociones y Publicaciones Universitarias-PPU.

Díaz Gómez, M. (2005). La educación musical en la escuela y el Espacio Europeo de Educación Superior. Revista Interuniversitaria de Formación del Profesorado, 19(1), 23-37.

Gutiérrez, P., Luengo, M.R., \& Casas, L.M. (2011). La formación de las maestras y la igualdad de oportunidades: ayer y hoy. Educatio Siglo XXI, 2(29), 333-352.

Infante, J. (2010). La reforma de los planes de estudio universitarios de la España democrática (19772000). Revista de Educación, 351, 259-282.

López Casanova, M.B. (2002). La música en el magisterio de las escuelas normales y su proyección en la primera enseñanza desde 1837 a 1930. Música y Educación. Revista Trimestral de Pedagogía Musical, 49, 29-44.

López García, N.J. (2015). Necesidades profesionales del profesorado especialista de música de los centros de educación primaria de Castilla-La Mancha (Tesis de doctorado no publicada). Universidad de Málaga: Departamento de Didáctica y Organización Escolar.

Lorenzo, J.A. (1995). Perspectiva histórica de la formación de los maestros en España (1370-1990). Revista Complutense de Educación, 2(6), 203-229.

Montero, M. (2010). El proceso de Bolonia y las nuevas competencias. Tejuelo, 9, 19-37.

Oriol, N. (2005). La música en las enseñanzas de régimen general en España y su evolución en el siglo XX y comienzos del XXI. LEEME. Revista de la Lista Electrónica Europea de Música en la Educación, 16. Recuperado de http://musica.rediris.es/leeme/revista/oriol05.pdf 